Check for updates

Cite this: RSC Adv., 2017, 7, 20281

Received 15th February 2017

Accepted 23rd March 2017

DOI: $10.1039 / c 7 r a 01908 d$

rsc.li/rsc-advances

\section{Tethering of rhBMP-2 upon calcium phosphate cement via alendronate/heparin for localized, sustained and enhanced osteoactivity $\dagger$}

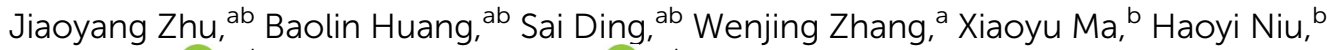 \\ Yuan Yuan (iD *ab and Changsheng Liu (iD *ab
}

Localized, continuous and effective osteogenic stimulation to defected sites is still a great challenge for recombinant human bone morphogenetic protein-2 (rhBMP-2) in clinical bone regeneration. In this study, a novel delivery system was engineered to tether rhBMP-2 onto the surface of calcium phosphate cement (CPC) based on the high affinity between alendronate and CPC, as well as the strong binding of heparin and rhBMP-2. Alendronate was first grated to heparin via the EDC/NHS reaction and then the resultant alendronate-heparin $(\mathrm{AH})$ was adsorbed onto the CPC surface. RhBMP-2 was further anchored onto the $\mathrm{CPC}-\mathrm{AH}$ surface. The results from in vitro release and in vivo fluorescence-labeled traces all indicated that the AH-tethered rhBMP-2 exhibited a more stable and stronger adherence to the CPC surface than the CPC-adsorbed and heparin-anchored ones. Moreover, based on the results of the alkaline phosphatase (ALP) activity in skeletal myoblasts (C2C12) in vitro and osteogenic efficacy in vivo, it could be seen that rhBMP-2-induced osteogenic bioactivity was also significantly enhanced on the $\mathrm{CPC}-\mathrm{AH}$ surface. These results demonstrated that the tethering of rhBMP-2 onto calcium phosphate surface via $\mathrm{AH}$ presented an effective method to achieve a localized and sustained exposure to targeted cells, and consequently to promote bone regeneration.

\section{Introduction}

In recent years, the remedy to severe bone trauma associated with bone loss, critical-sized bone defects, delayed union, or nonunion still presents a critical challenge in clinics. ${ }^{\mathbf{1 , 2}}$ For regeneration of these formidable defects, autografting remains the gold standard in orthopedic surgery due to its excellent osteoinductive and osteoconductive capabilities. ${ }^{3}$ However, the current available bone substitutes, unfortunately, often exhibit limited "osteoinductivity", resulting in undesirable therapeutic efficacy. To settle this issue, one of the most efficient and promising strategies is to incorporate/immobilize growth factors. ${ }^{4,5}$ Among these, recombinant human bone morphogenetic protein-2 (rhBMP-2), a potent osteoinductive growth factor of the transforming growth factor- $\beta$ (TGF- $\beta$ ) super family, is considered as the most notable cytokine to enhance bone formation $^{6,7}$ and has been approved by the US Food and Drug Administration for clinical applications since 2002. It is

${ }^{a}$ Key Laboratory for Ultrafine Materials of Ministry of Education, East China University of Science and Technology, Shanghai 200237, PR China. E-mail:yyuan@ecust.edu.cn; liucs@ecust.edu.cn

${ }^{b}$ Engineering Research Center for Biomedical Materials of Ministry of Education, East China University of Science and Technology, Shanghai 200237, PR China

$\dagger$ Electronic supplementary information (ESI) available. See DOI: 10.1039/c7ra01908d demonstrated that rhBMP-2 is efficient in skeletal defects repair in association with biomaterials by the recruitment and osteoinductive differentiation of MSC, angiogenesis and new bone integration. ${ }^{8-11}$ To fully utilize the function of rhBMP-2 to induce bone formation, a series of biomaterial carriers have been explored to satisfy the applied requirements; for instance, biodegradable polymers, ${ }^{12,13}$ polyelectrolyte multilayer coatings, ${ }^{\mathbf{1 4}, 15}$ titanium, ${ }^{\mathbf{1 6 , 1 7}}$ hydrogels, ${ }^{\mathbf{1 8 , 1 9}}$ and inorganic nanoparticles. ${ }^{20,21}$ Unfortunately, so far, even the most simple and traditional strategy for rhBMP-2 delivery, namely the usage of a collagen sponge carrier approved by the FDA, ${ }^{22}$ often results in protein denaturation and relatively unsatisfactory loading or releasing, which can lead to a supra-physiological dosage applied in the defect area, ${ }^{7}$ and subsequently can result in offtarget severe side effects, such as the stimulation of bone resorption, edema, inflammatory reactions, and undue tissue response in the host. ${ }^{23,24}$ Therefore, the highly efficient delivery of rhBMP-2 with a lower dose as well as a localized and sustained release while maintaining desirable osteoinductive bioactivity remains a great challenge.

Substantial investigations have confirmed that, to attain maximal efficacy, rhBMP-2 should be loaded into a biomaterial matrix. ${ }^{\mathbf{8 , 2 5 , 2 6}}$ Meanwhile, as a member of the TGF- $\beta$ family, BMP2 first binds to the specific BMP-2 membrane receptor on the cell surface, and then initiates the downstream signal transduction pathways inside cells. ${ }^{27-29}$ Therefore, an ideal matrix 
should not only provide localized, long-term available rhBMP-2, but should also preserve proper presentation for desirable receptor binding to the targeted cells. The tethering concept was first developed from the term "juxtacrine signaling" coined by Massague' in 1990, and aims to imitate the cellular communication between the signaling molecules and receptors that are both anchored to the cell membrane to deliver signals to each other and to regulate the cell functions. ${ }^{30}$ In the past few years, various tethering-based delivery systems have been developed for preserving the biological activity of growth factors. For example, Stefanie M. Shiels et al. used PEG to tether BMP-2 onto the HAP surface and found excellent bone induction, vessel growth, and scaffold-bone contact in this composite scaffold. ${ }^{31}$ Besides, the bi-functional FN-OCN fusion protein was tethered to the biopolymer scaffold proposed by Jae Ho Lee et al., and the positive influence of protein immobilization on the adhesion and osteogenic differentiation of MSCs was proved..$^{32}$ Truthfully, compared to the delivery vehicles that transport the growth factors to defect areas and take effect by releasing them in the form of solution, tethering systems that focus on the immobilization of growth factors are more conducive to prolong the exposure to the target spot of a bioactive protein, reduce the burst release, and improve the incorporation between tissue and material. ${ }^{33}$ Moreover, the tethering strategy can reduce the interaction between the growth factor and substrate, which can thus ensure its original "free-like" form to a large extent, which contributes to maintaining the biological function and activity of the growth factor.

Inspired by these pioneering investigations and rationales, in this study, we endeavored to develop a new tethering system for rhBMP-2 delivery. Considering the wide application of calcium phosphate in clinics, here, calcium phosphate cement (CPC), with excellent biocompatibility, degradation properties, new bone formation promotion, and, most importantly, a good similarity to the composition of the mineral phase of bone, was chosen as the matrix. ${ }^{34,35}$ To achieve effective tethering of rhBMP-2 onto CPC, alendronate (Alen), a bisphosphonate that can tightly bind to CPC surface and inhibit bone resorption simultaneously, ${ }^{36}$ and heparin (Hep), a kind of highly sulfated glycosaminoglycan with wide application for immobilization of the positive proteins by electrostatic adsorption, ${ }^{37}$ were used. As shown in Fig. 1A, Hep and Alen were first combined through an acylation reaction and then anchored onto the surface of CPC via the intense interaction between the phosphate groups of Alen and the calcium ions of CPC. Finally, rhBMP-2 was further incorporated upon the CPC surface through electrostatic interaction between Hep and rhBMP-2. The enduring immobilization and effective biological activity of rhBMP-2 were demonstrated through in vitro and in vivo assessments. This kind of delivery system first loaded BMP-2 upon CPC through the tethering concept and so totally avoided the direct contact as well as chemical reaction between the carrier and protein, which is a meaningful improvement based on the previous work to effectively maintain the bioactivity of proteins. Therefore, this novel delivery system was proved to have potential in the field of bone tissue regeneration.

\section{Experimental section}

\section{Synthesis and characterization of alendronate and heparin} (AH)

AH was synthesized through a condensation reaction between the carboxyl of heparin and the amino group of alendronate (Fig. 1B). In detail, at first, $1 \mathrm{~g}$ heparin sodium was dissolved in PBS (50 mL, pH = 5.6), then $380 \mathrm{mg}$ carbodiimide (EDC) and $230 \mathrm{mg} N$-hydroxysuccinimide (NHS) were added into the heparin solution and allowed to react for $30 \mathrm{~min}$ at room temperature (RT). After sufficient carboxylation of heparin, $217 \mathrm{mg}$ alendronate (Alen) was introduced to the mixture and the agitation was continued for $24 \mathrm{~h}$ at RT. Next, the resulting solution was transferred into a dialysis bag $(\mathrm{MwCO}=7000)$, which was totally emerged in the ultrapure water under stirring for $24 \mathrm{~h}$, with the external ultrapure water changed every $12 \mathrm{~h}$ in order to remove any unreacted small molecule compounds. Finally, the alendronate-modified heparin ( $\mathrm{AH}$ ) was collected by centrifugation and stored at RT for the subsequent experiments after being vacuum lyophilized for $48 \mathrm{~h}$.

The successful fabrication of $\mathrm{AH}$ was characterized by Fourier transform infrared (FT-IR) spectrometry (Nicolet 6700, USA) using $\mathrm{KBr}$ pellets under ambient conditions and by nuclear magnetic resonance (NMR) spectroscopy (Bruker AVANCE III 600, Bruker Corporation, Switzerland) in $\mathrm{D}_{2} \mathrm{O}$.

\section{Preparation and functionalization of CPC}

The CPC disk sample was prepared by the pellet pressing method. Briefly, 0.2 g CPC powder (Rebone Biomaterials Co., Ltd. Shanghai, China) and $50 \mu \mathrm{L}$ deionized water were mixed in a plastic mortar, and then the mixture was stirred sufficiently to form a homogeneous paste. After that, the resulting paste was uniaxially pressed at $2 \mathrm{MPa}$ for $1 \mathrm{~min}$ in a stainless steel mold. Finally, the CPC disk-shaped pellets $10 \mathrm{~mm}$ in diameter and 2 $\mathrm{mm}$ in thickness ( $0.2 \mathrm{~g}$ per pellet) were formed and kept at $37^{\circ} \mathrm{C}$ and $100 \%$ relative humidity for $72 \mathrm{~h}$ in the oven. The hardened CPC samples were obtained by soaking in the ultrapure water for $24 \mathrm{~h}$, in order to wash off the unset powders from the surface. The porous CPC scaffolds for the in vivo experiments were obtained through the $\mathrm{NaCl}$ particle leaching procedure described in our previous study. ${ }^{38}$ All the scaffolds for the in vivo experiments were sterilized by damp-heat sterilization at $121^{\circ} \mathrm{C}$ and 1.21 MPa for $30 \mathrm{~min}$.

The AH was physically adsorbed onto the surface of the CPC sample via lyophilization. Briefly, the CPC was immersed in an AH solution of PBS $(\mathrm{pH}=7.4)$ with the concentration of $1 \mathrm{mg}$ $\mathrm{mL}^{-1}$. After $24 \mathrm{~h}$ incubation in the constant temperature orbital shaker incubator, the sample was washed with PBS twice in order to remove the unstable molecules on the surface. All the washed PBS were collected together to calculate the amount of $\mathrm{AH}$ adsorbed onto the CPC surface by using the subtraction method. Finally, CPC-AH was obtained by vacuum lyophilization. In a similar method, the CPC-heparin was well prepared.

The successful adsorption of $\mathrm{AH}$ on the CPC sample was analyzed by using a toluidine blue assay. ${ }^{39}$ Toluidine blue (TB) 
A
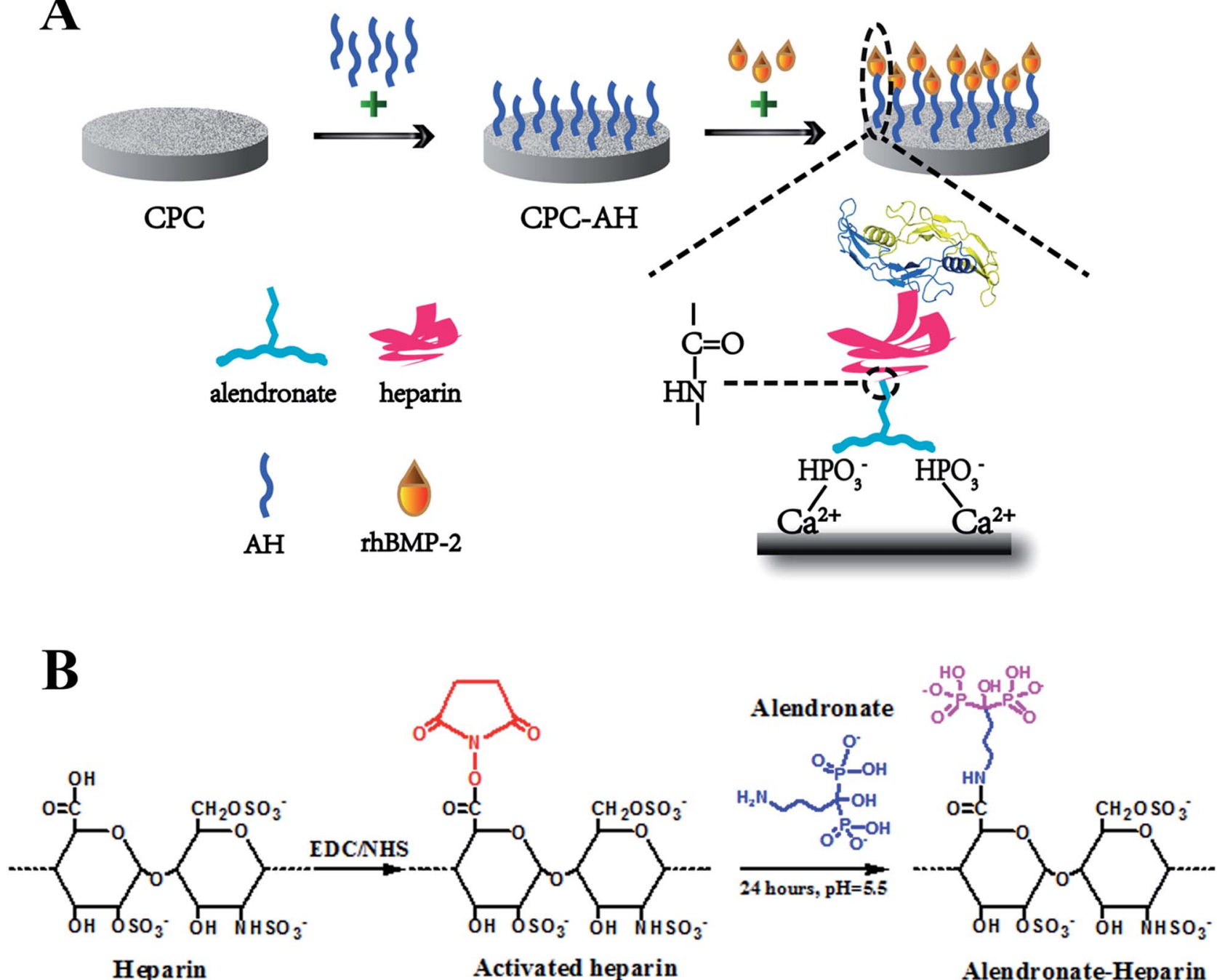

Alendronate-Heparin

(AH)

Fig. 1 (A) Schematic representation of the rhBMP-2 tethering system based on AH-modified CPC. AH was grafted onto the surface of CPC through the tight binding between alendronate and CPC, and then rhBMP-2 was tethered by the interaction with heparin. (B) Synthetic scheme for synthesis of an $\mathrm{AH}$ molecule via the condensation reaction between heparin and alendronate.

was used to detect the substance containing polyanion groups, such as heparin, by a color-changeable reaction from blue to purple. First, TB was used in the qualitative detection. The toluidine blue solution was prepared as follows: $0.5 \mathrm{mg}$ of toluidine blue and $20 \mathrm{mg}$ of $\mathrm{NaCl}$ were added to the $\mathrm{HCl}$ solution $(10 \mathrm{~mL} 0.01 \mathrm{M})$. Then, four different groups, namely CPC, CPC-alendronate (CPC-Alen), CPC-heparin (CPC-Hep), and the CPC-AH samples were immersed completely in toluidine blue solution, respectively, and then incubated for $30 \mathrm{~min}$ at RT. Finally, the color differences between the dyed samples were observed using a digital camera (Nikon D5000, Japan).

The surface morphology of the CPC/CPC-AH samples were observed by scanning electron microscopy (SEM, S-4800, Hitachi, Japan), and the surface distribution of the relative elements was analyzed by energy dispersive X-ray spectra (EDS, QUANTAX 400-30, Germany).

\section{Adsorption and release of AH from CPC}

The adsorption ability of AH to the CPC surface was first investigated. The CPC was soaked in $1 \mathrm{mg} \mathrm{mL}^{-1} \mathrm{AH}-\mathrm{PBS}$ solution, and the amount of adsorbed $\mathrm{AH}$ was calculated by collecting the AH solution at scheduled time points $(1,3,7,12,24$ h) and analyzed by using the colorimetric toluidine blue assay. Briefly, $2 \mathrm{~mL}$ AH-PBS was mixed with $3 \mathrm{~mL}$ toluidine blue through a vortex vibrator for $30 \mathrm{~min}$. After the complete reaction, $3 \mathrm{~mL}$ hexane was added to the solution, followed by sufficient oscillation to extract the TB-AH complex. Finally, the upper layer was discarded and $200 \mu \mathrm{L}$ of the solution was collected from the lower layer to determine the optical density using an enzyme-linked immunoadsorbent assay plate reader (SPECTRAmax 384, Molecular Devices, USA) at $630 \mathrm{~nm}$. The heparin adsorption ability was determined by a similar method.

Next, the release behavior of AH from the CPC surface was investigated by immersing the $\mathrm{CPC}-\mathrm{AH} / \mathrm{CPC}-\mathrm{Hep}$ samples in 10 
$\mathrm{mL}$ PBS $(\mathrm{pH}=7.4)$. The $5 \mathrm{~mL}$ release medium was removed at selected time intervals $(1 \mathrm{~h}, 3 \mathrm{~h}, 7 \mathrm{~h}, 12 \mathrm{~h}, 1 \mathrm{~d}, 3 \mathrm{~d}, 7 \mathrm{~d}, 14 \mathrm{~d}, 21$ $\mathrm{d}$ and $28 \mathrm{~d}$ ) and then replaced by an equal volume of fresh PBS. Finally, the released $\mathrm{AH}$ was calculated according to the $\mathrm{AH}$ concentration standard curve using the toluidine blue assay. The CPC-Hep sample was also evaluated at the same condition as a control. The experiments were run in triplicate per sample.

\section{Tethering and release of rhBMP-2 onto/from CPC-AH}

After modification, the saturated adsorption ability of the composite materials was first evaluated by rhBMP-2 ELISA Kit (PeproTech, Rocky Hill, NJ) using an immersion method. In brief, the CPC, CPC-Hep, and CPC-AH samples were immersed in $1 \mathrm{~mL}$ rhBMP-2 $\left(12.5,25,50,100,200\right.$, and $\left.400 \mu \mathrm{g} \mathrm{mL}{ }^{-1}\right)$ for $6 \mathrm{~h}$ under $4{ }^{\circ} \mathrm{C}$, respectively. Then, the rhBMP-2 solutions were collected and the adsorbed samples were washed twice with PBS to remove the unstable rhBMP-2 on the surface.

In the rhBMP-2 adsorption experiment, rhBMP-2 in acetic acid solution was diluted and dropped onto the CPC, CPC-Hep, and $\mathrm{CPC}-\mathrm{AH}$ samples, respectively ( $2 \mu \mathrm{g}$ per sample), and all samples were kept for $6 \mathrm{~h}$ under $4{ }^{\circ} \mathrm{C}$ for full adsorption. After being lyophilized, the final CPC/rhBMP-2, CPC-Hep/rhBMP-2, and CPC-AH/rhBMP-2 samples were obtained and could be long-term preserved at $4{ }^{\circ} \mathrm{C}$ before use.

The in vitro protein release was studied by suspending the CPC-Hep/rhBMP-2 or CPC-AH/rhBMP-2 sample in PBS $(\mathrm{pH}=$ $7.4)$ in a constant temperature incubator shaker $\left(37{ }^{\circ} \mathrm{C}, 100\right.$ $\mathrm{rpm})$. At pre-determined time intervals $(1,3,7,12,24,72$, and $168 \mathrm{~h}$ ), all the released solution was collected, and an identical volume of fresh PBS was added. The concentration of rhBMP-2 for each collected sample were measured by a rhBMP-2 ELISA kit to calculate the released amount.

\section{Cell culture}

C2C12 was purchased from the American Type Culture Collection (ATCC) and incubated in Dulbecco's modified Eagle's medium (DMEM) supplemented with 10\% fetal bovine serum (FBS) at $37^{\circ} \mathrm{C}$ and $100 \%$ humidity with $5 \% \mathrm{CO}_{2}$. The cell density was calculated by using a cytometer after being detached using $0.25 \%$ trypsin $/ 0.03 \%$ ethylene diamine tetraacetic acid (EDTA). All the cell culture reagents were obtained from Sigma-Aldrich, Saint Louis, MO, USA. For the rhBMP-2 group, rhBMP-2 with a concentration of $0.4 \mu \mathrm{g} \mathrm{mL}{ }^{-1}$ was added in the origin media.

\section{MTT assay}

The methyl thioazolyl tetrazolium (MTT, Sigma-Aldrich, USA) assay was performed to investigate the effect of AH concentration on the cell viability of C2C12. Briefly, $1 \mathrm{mg} \mathrm{mL}^{-1} \mathrm{AH}-\mathrm{PBS}$ solution was prepared by sterile filtration $(0.2 \mu \mathrm{m})$. C2C12 cells were seeded at a density of $3 \times 10^{4}$ per well in a 24 -well plate, with the addition of AH-PBS solution under different concentration $\left(0.5,1,10,50\right.$, and $100 \mu \mathrm{g} \mathrm{mL}{ }^{-1}$, respectively). In the control group, the cells were cultured directly on the 24-well plate without $\mathrm{AH}$. The rhBMP-2 group was examined with a 0.4 $\mu \mathrm{g} \mathrm{mL} \mathrm{L}^{-1}$ rhBMP-2 addition. After 3 day incubation at $37^{\circ} \mathrm{C}$ and $100 \%$ humidity with $5 \% \mathrm{CO}_{2}$, MTT was added to each well and incubated at the same condition for $4 \mathrm{~h}$. Then, the supernatant was removed and mixed with dimethyl sulfoxide (DMSO) for 15 min under vibration. Finally, the optical density (OD) of the chromophore was measured at $492 \mathrm{~nm}$ by an enzyme-linked immuneadsorbent assay plate reader. Results were reported as the percentage ratio of $\mathrm{OD}_{\text {sample }} / \mathrm{OD}_{\text {control }} \times 100 \%(n=4)$.

\section{Alkaline phosphatase (ALP) activity assay}

The effect of $\mathrm{AH}$ concentration on the differentiation ability of C2C12 was investigated with the ALP activity assay. C2C12 cells were seeded with AH-PBS solution of different concentrations $\left(0.5,1,10,50\right.$, and $100 \mu \mathrm{g} \mathrm{mL}{ }^{-1}$, respectively) at a density of $3 \times$ $10^{4}$ cells per sample in a 24 -well plate and incubated in DMEM. After 3 day treatment, about $200 \mu \mathrm{L}$ of 1\% Nonidet P-40 (NP-40) solution was added into each well and then incubated for $2 \mathrm{~h}$ at $37^{\circ} \mathrm{C}$. Then, $50 \mu \mathrm{L}$ of cell lysate was transferred to 96 -well plates and $100 \mu \mathrm{L}$ of $p$-nitrophenylphosphate (PNPP-Na, $1.0 \mathrm{mg} \mathrm{mL}^{-1}$ ) was added and allowed to incubate for a further $15 \mathrm{~min}$ at $37^{\circ} \mathrm{C}$. After incubation, $50 \mu \mathrm{L}$ of $0.1 \mathrm{M} \mathrm{NaOH}$ was added to each well for termination. The optical density (OD) value was determined at the wavelength of $405 \mathrm{~nm}$ using an enzyme-linked immunoadsorbent assay plate reader. The total protein content was quantified with bicinchoninic acid (BCA) assay kits (Beyotime, Shanghai, China).

The osteogenic differentiation ability of the functionalized CPC samples was evaluated by detecting the ALP activity. C2C12 cells were seeded on CPC/rhBMP-2, CPC-Hep/rhBMP-2, and CPC-AH/rhBMP-2 samples at an initial density of $2 \times 10^{4}$ per well in a 24-well plate. The ALP activity and total protein content were measured after 3 day and 7 day treatment.

ALP staining assay was also processed to determine the effect of the loaded samples on the osteogenic differentiation of the cells. After fixation of $\mathrm{C} 2 \mathrm{C} 12$ cells in $2.5 \%$ glutaraldehyde for $10 \mathrm{~min}$ on ice, the cells were incubated in $200 \mu \mathrm{L} \mathrm{BCIP/NBT}$ working solution (Alkaline Phosphatase Stain Assay Kit, Beyotime, Shanghai, China) at RT. After $30 \mathrm{~min}$, the ALP staining working solution was removed to stop the reaction and all the samples were rinsed twice with PBS. The stained cells were visualized using an inverted light microscope (TE2000U, Nikon Corp., Japan).

\section{In vivo experiments}

This study was performed in strict accordance with the NIH guidelines for the care and use of laboratory animals (NIH Publication no. 85-23 Rev. 1985) and was approved by the Institutional Animal Care and Use Committee of National Tissue Engineering Center (Shanghai, China). Thirteen male C57BL/6 mice (five weeks old, Jiesijie Inc. Shanghai, China) were anesthetized by intraperitoneal injection of the pentobarbital. The CPC/rhBMP-2 and CPC-AH/rhBMP-2 scaffolds were implanted symmetrically into the left and right thigh muscle pouches of each mouse. Among these, three mice were used for the in vivo imaging and the rest were allocated equally into week 2 and 4 time points for ectopic bone formation. 


\section{In vivo release of rhBMP-2 from CPC}

The release behavior of rhBMP-2 from the CPC and CPC-AH scaffolds in vivo was evaluated by in vivo imaging in animals. The rhBMP-2 was labeled with FITC first, and then was adsorbed onto the surfaces of CPC (as the control) and the CPC-AH samples by the same procedure as in the in vitro experiment. At the scheduled time points after implantation $(0,3,6,12,24,72$, and $168 \mathrm{~h}$ ), the mice were anesthetized and scanned using a Kodak multi-model imaging system (Carestream Health, Inc., USA, excitation: $480 \mathrm{~nm}$, emission: $553 \mathrm{~nm}$ ) to monitor the change of rhBMP-2 distribution. The fluorescence intensity of the image was analyzed using a Carestream molecular imaging system. The X-ray images were established using a 35 KVP X-ray beam.

\section{Ectopic bone formation}

In vivo ectopic bone formation of the CPC/rhBMP-2 and CPCAH/rhBMP-2 samples in the thigh muscle pouches of the mice was investigated at 2 weeks and 4 weeks. The rhBMP-2 dosage and adsorption procedure were kept the same as in the in vitro experiments. At 2 and 4 weeks after surgery, the mice ( $n=5$ for each group) were sacrificed with an overdose of pentobarbital and the implants were harvested to investigate the ectopic bone formation. All the retrieved specimens were detached from the surrounding soft tissue and weighed for wet content.

\section{Synchrotron radiation-based micro-computed tomography (SR $\mu \mathrm{CT}$ ) imaging}

The bone specimens were imaged by synchrotron radiationbased micro-computed tomography (SR $\mu \mathrm{CT})$ measurements at beamline BL13W of the Shanghai Synchrotron Radiation Facility (SSRF, Shanghai, China) to determine the new bone morphology and volume. A monochromatic beam with an energy of $23 \mathrm{keV}$ was used and the distance of the sample to the detector was $0.35 \mathrm{~m}$. In the current study, a VHR detector (Photonic Science Company, UK) with a pixel size set to $9 \mu \mathrm{m}$ was used to record the images. 1200 projections within an angular range of $180^{\circ}$ were taken and the exposure time amounted to $8 \mathrm{~ms}$ per projection. The reconstructed 3D images and percentage of new bone volume relative to the total volume were analyzed by Amira 6.0.1 software (Visage Imaging Company, Australia).

\section{Histological evaluation}

The histological analysis was assigned for further investigation with the retrieved specimens. After fixation with $4 \%$ neutral buffered formalin for $48 \mathrm{~h}$, the ectopic bone samples were decalcified in $12.5 \%$ EDTA, dehydrated in a graded series of alcohols, and embedded in paraffin. After that, serial $4 \mu \mathrm{m}$ thick sections were stained with hematoxylineosin (HE) and Masson's trichrome staining, respectively, and then visualized under a light microscope (Nikon TE2000U, Japan).

\section{Statistical analysis}

All the data were expressed with the mean standard deviation (SD) and analyzed using one-way ANOVA followed by Tukey's post hoc test; a $p$ value $<0.05$ was considered statistically significant.

\section{Results}

\section{Synthesis and characterization of $\mathrm{AH}$}

Alendronate-heparin $(\mathrm{AH})$ was obtained through a typical acylation reaction, as shown in Fig. 1B, and evidence for the successful synthesis of AH was directly confirmed from FT-IR, ${ }^{1} \mathrm{H}-\mathrm{NMR}$, and ${ }^{13} \mathrm{C}-\mathrm{NMR}$ spectroscopy. As shown in Fig. 2A, for the spectrum of heparin, a peak appearing at $1621 \mathrm{~cm}^{-1}$ was assigned to the stretching vibration of $\mathrm{C}=\mathrm{O}$ of carboxyl, and the absorption bands at $1240 \mathrm{~cm}^{-1}$ and $1027 \mathrm{~cm}^{-1}$, corresponding to asymmetric and symmetric stretching vibration of $-\mathrm{SO}_{3}-$
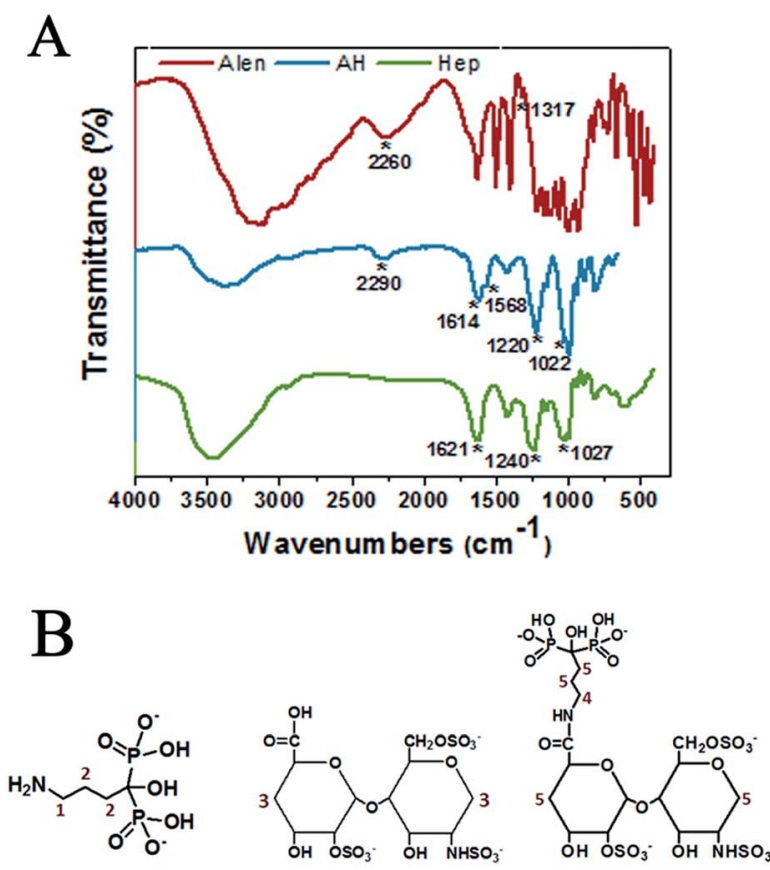

Alendronate Heparin $\mathrm{AH}$

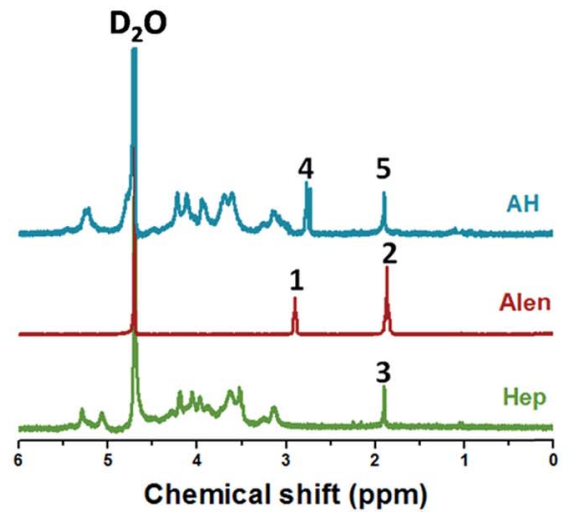

Fig. 2 Characterization of $A H$ molecule. (A) FT-IR spectra and (B) ${ }^{1} H$ NMR spectra of Alen, Hep, and $\mathrm{AH}$. 
,respectively, were also clearly observed. Similarly, typical absorption bands of $\mathrm{C}=\mathrm{O}$ of carbonyl (around $1614 \mathrm{~cm}^{-1}$ ) and$\mathrm{SO}_{3}-$ groups (around 1220 and $1022 \mathrm{~cm}^{-1}$ ) were also observed in the spectrum of $\mathrm{AH}$. As for the spectrum of alendronate, a representative absorption band around $2260 \mathrm{~cm}^{-1}$, corresponding to the stretching vibration of the $(\mathrm{O}=\mathrm{P}-\mathrm{OH}-\mathrm{O})$ group, was observed, which was also exhibited in the spectrum of $\mathrm{AH}$ at around $2290 \mathrm{~cm}^{-1}$. Additionally, besides these characteristic peaks of the common groups, a specific absorption band in the spectrum of $\mathrm{AH}$ was also captured at $1568 \mathrm{~cm}^{-1}$ that represented the stretching vibration of $\mathrm{C}-\mathrm{N}$ and the bending vibration of $\mathrm{N}-\mathrm{H}$ (overlapped) from the amide group, and differently, the stretching vibration of $\mathrm{C}-\mathrm{N}$ from the amine of alentronate appeared at $1317 \mathrm{~cm}^{-1}$. Therefore, the successful synthesis of AH was demonstrated by FT-IR, while the ${ }^{1} \mathrm{H}-\mathrm{NMR}$ and ${ }^{13} \mathrm{C}$ NMR analyses also provided essential further proof.

As illustrated in Fig. 2B, an intensive peak at $4.8 \mathrm{ppm}$ was ascribed to the $\mathrm{D}_{2} \mathrm{O}$ solvent. The resonance signal at $1.90 \mathrm{ppm}$ (peak 3) was assigned to the $-\mathrm{CH}_{2}-$ (3) in heparin, while the signal at $2.90 \mathrm{ppm}$ (peak 1) was ascribed to methylene protons close to the amide group in alendronate, and the peaks at 1.83$1.9 \mathrm{ppm}$ (peak 2) corresponded to the other $-\mathrm{CH}_{2}-(2)$. The similar peaks appearing in AH were at $2.77 \mathrm{ppm}$ (peak 4), which referred to the $-\mathrm{CH}_{2}-$ (4) from alendronate, while the overlapped peak of $-\mathrm{CH}_{2}-$ (5) from alendronate and heparin, respectively, was at 1.87-1.96 ppm (peak 5). Furthermore, the chemical shifts of the $-\mathrm{CH}_{2}-(4)$ at $2.77 \mathrm{ppm}$ in $\mathrm{AH}$ might be influenced by the grafted heparin molecule. Additionally, the ${ }^{13} \mathrm{C}-\mathrm{NMR}$ analysis disclosed further details of these molecules and the successful synthesis of $\mathrm{AH}$, which are given in the ESI (Fig. S1†).

\section{Adsorption of AH on the surface of CPC}

The successful adsorption of AH to the CPC surface was estimated using a colorimetric toluidine blue assay. All the CPC samples with/without modification were white disks. As shown in Fig. 3A, the colors of the CPC-AH and CPC-Hep samples turned into purple after dying with toluidine blue, while the pure CPC sample remained white as before.

To gain a more intuitive understanding of the distribution of the essential elements on the CPC-AH and CPC surfaces, surface area scanning and mapping of the relative elements were performed by energy dispersive X-ray spectra (EDS) (Fig. 3B). A good distribution of $\mathrm{Ca}, \mathrm{P}$, and $\mathrm{O}$ (shown as red, green, and blue dots, respectively) was observed in the resulting map. Specifically, CPC-AH exhibited the extra elements of C, N, and $S$, which were attributed to the $\mathrm{AH}$ section. In addition, the morphology of the CPC-AH sample displayed indistinctive difference from the CPC sample (Fig. 3C).

The interaction between $\mathrm{AH}$ and the $\mathrm{CPC}$ surface was investigated to estimate the adsorption ability of AH on CPC. As depicted in Fig. 3D, compared to pure heparin, the alendronatemodified heparin was more facile to be adsorbed onto the CPC surface due to the intense electrostatic interaction between alendronate and CPC. Obviously, AH was adsorbed on the CPC surface much faster, especially in the early stage. When merged in $\mathrm{AH}$ solution for $1 \mathrm{~h}$, almost $50 \mu \mathrm{g} \mathrm{AH}$ was adsorbed onto the CPC surface, approximately 5 -fold more than the heparin counterpart, which was only $10 \mu \mathrm{g}$. For both molecules, the adsorption process nearly reached balance after incubation for $12 \mathrm{~h}$. Finally, the maximum adsorption amount of $\mathrm{AH}$ onto the CPC surface was $98 \mu \mathrm{g}$, which was almost double that of heparin. Next, the stability of the adsorbed AH on the CPC
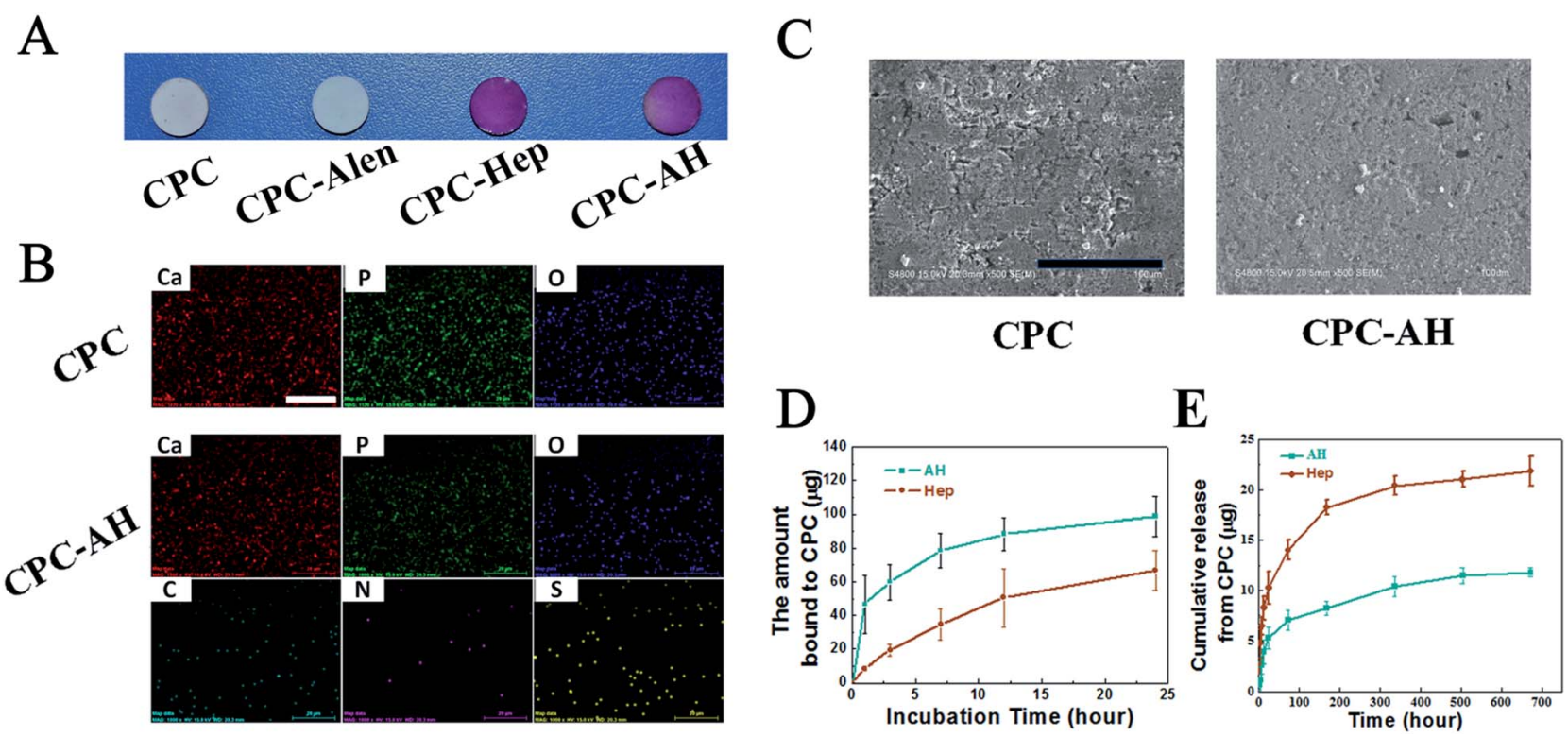

Fig. 3 Characterization, surface morphology, and binding capability of the CPC-Hep and CPC-AH samples. (A) Different samples detected by colorimetric toluidine blue assay. (B) EDS mapping of the essential elements distribution on the surfaces of the CPC and CPC-AH samples. The white scale bar is $20 \mu \mathrm{m}$. (C) Surface morphology of CPC and CPC-AH by SEM. The black scale bar is $100 \mu \mathrm{m}$. (D) The adsorption and (E) release profiles of Hep and $\mathrm{AH}$ from the CPC sample. 
surface was further investigated (Fig. 3E). The release curve of $\mathrm{AH} /$ heparin detected in PBS solution exhibited that $\mathrm{AH}$ was more durable compared with heparin existing on the surface of CPC. After 28 days, the accumulative release of AH was only 10 $\mu \mathrm{g}$ (of $98 \mu \mathrm{g}$ ), while that of heparin was $21.8 \mu \mathrm{g}$ (of $62 \mu \mathrm{g}$ ).

All the data indicated that $\mathrm{AH}$ had a faster adsorption and slower release rate than heparin on the CPC surface, which could be ascribed to the strong binding affinity of alendronate to the CPC substrate.

\section{Effect of AH on cell viability and differentiation}

In order to explore the cellular response of $\mathrm{AH}$, the viability of C2C12 cells cultured with AH under different concentrations for 3 days was investigated using the MTT assay. As shown in Fig. 4A, the cell viability distinctly decreased with the increase in AH concentration, while the group with the addition of rhBMP2 showed a similar trend. The influence of the concentration of $\mathrm{AH}$ on the cell viability displayed no significant difference between the groups with/without rhBMP-2. Specifically, the cell viability declined by $50 \%$ compared to the control group with an $\mathrm{AH}$ addition of $50 \mu \mathrm{g} \mathrm{mL} \mathrm{m}^{-1}$.

Additionally, the effect of AH on the ALP activity with/without rhBMP-2 is demonstrated in Fig. 4B. Obviously, compared with the cells in DMEM (control group), $\mathrm{AH}$ alone did not stimulate an increase in ALP activity under any concentration. However, in the rhBMP-2-added groups, an AH dose-dependent manner on rhBMP-2-induced ALP activity was revealed, and the result showed that ALP activity was enhanced with the addition of $\mathrm{AH}$ and furthermore that the highest ALP activity was detected under the $\mathrm{AH}$ concentration of $10 \mu \mathrm{g} \mathrm{mL}{ }^{-1}$. Interestingly, with further $\mathrm{AH}$ addition into the culture system, the ALP expression was attenuated continually and even became lower than the control group with the addition of $50 \mu \mathrm{g} \mathrm{mL}{ }^{-1}$.

The results suggest that the cumulative released amounts of $\mathrm{AH}$ from CPC at any time point during 28 days $\left(1.18 \mu \mathrm{g} \mathrm{mL}{ }^{-1}\right.$ at 28 days) all had no obvious negative effect on cell viability; in particular, low-dose AH obviously enhanced the rhBMP-2induced osteogenic differentiation.

\section{Tethering and in vitro release kinetics of rhBMP-2}

To investigate the adsorption ability of rhBMP-2 onto the CPC and CPC-HA/heparin samples, the isothermal adsorption at
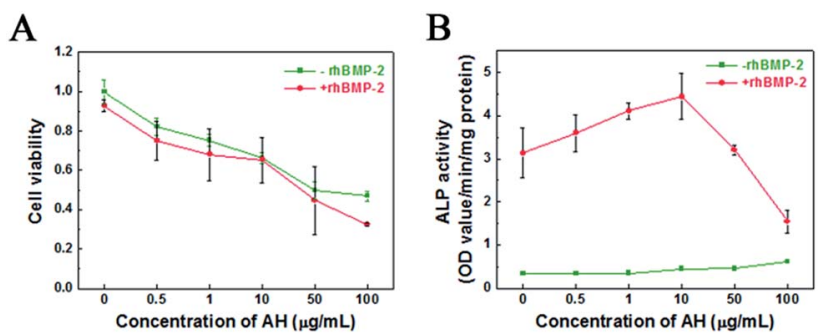

Fig. 4 Effect of $\mathrm{AH}$ on cell viability and differentiation. (A) Cell viability on $\mathrm{AH}$ with different concentrations after 3 days determined by MTT assay. (B) ALP activity of $\mathrm{C} 2 \mathrm{C} 12$ cells at 3 days induced by rhBMP-2 in the presence of $\mathrm{AH}$. (A dose of $0.4 \mu \mathrm{g} \mathrm{mL}^{-1}$ for rhBMP-2 was applied.) various concentrations of rhBMP-2 was detected. ${ }^{40}$ As shown in Fig. 5A, all the groups exhibited a similar adsorption behavior, and the adsorbed curves were fitted with the Langmuir adsorption equation as following:

$$
q_{\mathrm{e}}=\frac{Q_{\mathrm{m}} K_{\mathrm{eq}} c}{1+K_{\mathrm{eq}} c}
$$

where $q_{\mathrm{e}}$ represents the binding capacity of rhBMP-2 adsorbed onto the surfaces of materials at equilibrium, $c$ is the concentration of rhBMP-2, $K_{\text {eq }}$ is the equilibrium binding constant, and $Q_{\mathrm{m}}$ represents the maximum binding capacity of rhBMP-2. The relative parameters are listed in Table 1.

From the isothermal adsorption curve, we discovered that with the original concentration of rhBMP-2 enhanced, the adsorbed amount of rhBMP-2 was increased rapidly and finally reached balance. The maximum adsorption amount of rhBMP-2 exceeded $10 \mu \mathrm{g}$ in all the groups, which ensured that the amount of rhBMP-2 in the following experiments was totally located in the saturated adsorption range, and was kept stable on the sample surface.

Following the loading experiment, the release kinetics of the rhBMP-2 was evaluated by immersing the rhBMP-2-tethered CPC-Hep or CPC-AH samples into PBS solution $(\mathrm{pH}=7.4)$. As indicated by the release curve in Fig. 5B, for both samples, the profiles exhibited a typical two-stage release kinetics: an initial burst occurring during the first $24 \mathrm{~h}$ and then a slowly sustained release during the rest of the period. However, the initial release rate was drastically reduced for $\mathrm{CPC}-\mathrm{AH} / \mathrm{rhBMP}$ compared to CPC-Hep/rhBMP, and the cumulative rhBMP-2 release from CPC-Hep/rhBMP was greater than that of CPC$\mathrm{AH} / \mathrm{rhBMP}$ at all the evaluated periods. Under the condition of the same amount of adsorption, it was calculated that approximately $60 \%$ of rhBMP-2 was released in the first $24 \mathrm{~h}$ from CPC-Hep/rhBMP, while the amount of rhBMP-2 released from
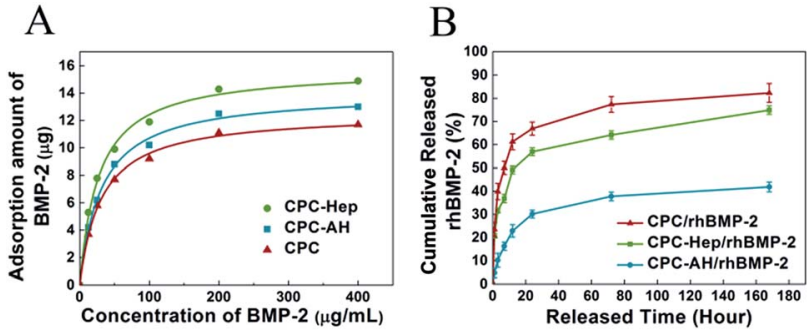

Fig. 5 (A) Isothermal adsorption curves of rhBMP-2 at various concentrations on the surfaces of the CPC, CPC-Hep, and $\mathrm{CPC}-\mathrm{AH}$ samples. (B) The in vitro cumulative release profiles of rhBMP-2 from the CPC, CPC-Hep, and CPC-AH samples in PBS.

Table 1 Fitting parameters for the binding of rhBMP-2 onto the CPC, CPC-Hep, and CPC-AH samples

\begin{tabular}{llll}
\hline & CPC & CPC-Hep & CPC-AH \\
\hline$Q_{\mathrm{m}}(\mu \mathrm{g})$ & $12.54 \pm 0.22$ & $15.80 \pm 0.35$ & $14.03 \pm 0.29$ \\
$K_{\mathrm{eq}}$ & $0.033 \pm 0.002$ & $0.037 \pm 0.003$ & $0.032 \pm 0.002$ \\
$R^{2}$ & 0.997 & 0.995 & 0.996
\end{tabular}


CPC-AH/rhBMP was only $30 \%$ during the same time. After 7 days, almost $75 \%$ of the originally tethered rhBMP-2 was released from the CPC-Hep/rhBMP sample, and the accumulative release retained an increasing trend over time. In contrast, a relatively stable release speed was displayed and the amount of accumulative release was sustained around $40 \%$.

These results implied that the rhBMP-2-tethered CPC-AH sample presented a slower and more sustained release behavior over the entire testing period in comparison to CPC-Hep/ rhBMP.

\section{Osteogenic bioactivity of rhBMP-2 tethered upon CPC-AH}

ALP activity, an early marker indicating cell differentiation Toward an osteoblast, was used to determine the bioactivity of rhBMP-2 to induce osteogenic differentiation in the $\mathrm{C} 2 \mathrm{C} 12$ cell line. ALP staining (Fig. 6A) at 7 days showed that the most intense ALP staining was found for cells cultured on $\mathrm{CPC}-\mathrm{AH} /$ rhBMP-2, revealing that rhBMP-2 retained the most prominent osteogenic bioactivity in this group. The groups without the addition of rhBMP-2 barely displayed ALP activity. In addition, the results of the quantitative assay demonstrated a similar trend (Fig. 6B). In the early time point (3 days), compared with the CPC/rhBMP-2 and CPC-Hep/rhBMP-2

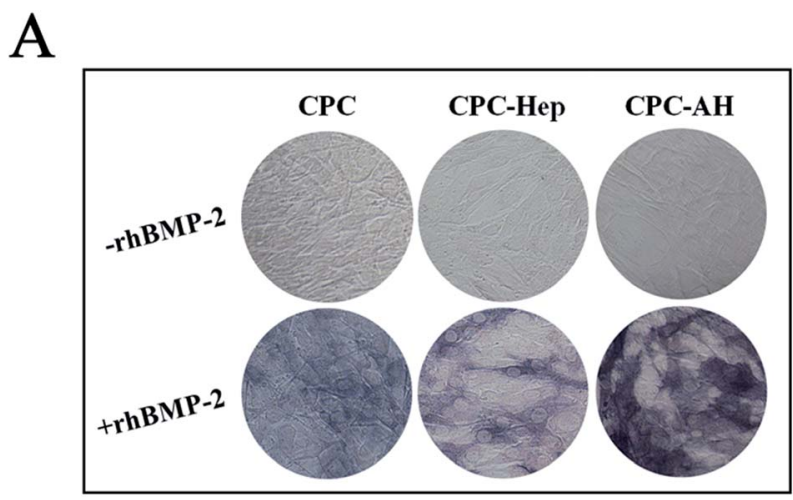

B

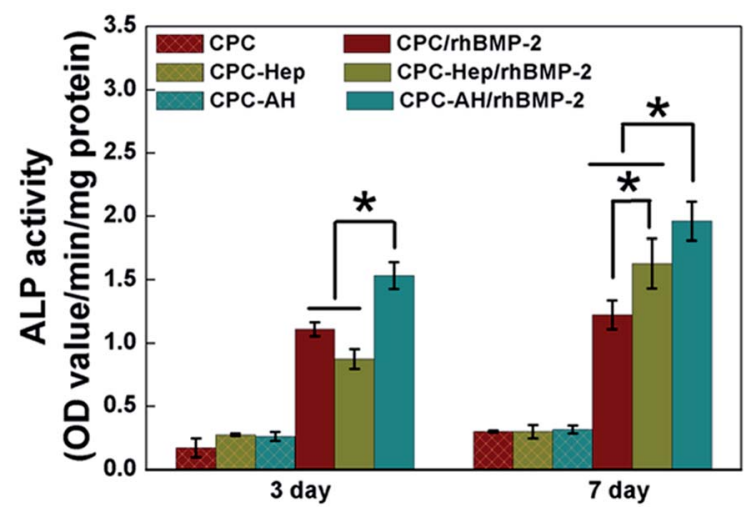

Fig. 6 Effect of rhBMP-2 immobilized upon CPC, CPC-Hep, and $\mathrm{CPC}-\mathrm{AH}$ on the osteogenic differentiation of $\mathrm{C} 2 \mathrm{C} 12 \mathrm{~s}$. (A) ALP staining of $\mathrm{C} 2 \mathrm{C} 12 \mathrm{~s}$ cultured on different samples for 7 days. (B) ALP activity of C2C12s cultured on different samples at days 7 and 14 of incubation. Results are the mean \pm standard deviation $(n=4),{ }^{*} p<0.05$. groups, only the $\mathrm{CPC}-\mathrm{AH} / \mathrm{rhBMP}-2$ group enhanced the ALP activity. At day 7 after cell seeding, the ALP activities of the functionalized CPC-loaded groups, especially CPC-AH/rhBMP2 , were significantly higher than that of CPC/rhBMP-2. There was no ALP activity expressed in any group without rhBMP-2 at days 3 and 7.

Based on the above results, the $\mathrm{AH}$ molecule adsorbed onto the surface of CPC sample facilitated the bioactivity of rhBMP-2 to induce osteogenic differentiation through effective tethering and by concentrating rhBMP-2 around the sample surface, which accelerated osteogenesis in early stage.

\section{In vivo release of tethered rhBMP-2 upon CPC-AH}

The real-time in vivo imaging experiment was performed to investigate the in vivo release behavior of the tethered FITClabeled rhBMP-2 on the scaffolds (as shown in Fig. 7A).

Immediately after implantation of the CPC/rhBMP- 2 and CPC-AH/rhBMP-2 samples, the fluorescence emitted from the labeled rhBMP-2 was facilely visualized and reached the maximum value locally. After $3 \mathrm{~h}$ post-operation, the luminescent signal from CPC/rhBMP-2 (left) gradually weakened and the center intensity then completely disappeared after $12 \mathrm{~h}$. However, the CPC-AH/rhBMP-2 sample (right) sustained the significant intensity of the center signal until $72 \mathrm{~h}$. The weak luminescent signal was found in the head, tail, and limbs after $6 \mathrm{~h}$ due to the relatively high metabolic rate in those parts. In addition, the beginning values of the mean fluorescent intensity (MFI) were similar for the CPC/rhBMP-2 and CPC-AH/rhBMP-2 samples, while the MFI of CPC-AH/rhBMP-2 was twice that of $\mathrm{CPC} / \mathrm{rhBMP}-2$ at $3 \mathrm{~h}$ and persistently more intense than that of CPC/rhBMP-2 during $168 \mathrm{~h}$ (as shown in Fig. 7B).

The data also revealed that the $\mathrm{CPC}-\mathrm{AH}$ scaffold could effectively confine rhBMP-2 around the vicinity while releasing it, instead of the random diffusion that was shown in the CPC sample. This tethering character of CPC-AH supported the local immobilization and sustainably high concentration of rhBMP2 , which availably avoided the invalid loss of rhBMP-2 and consequently enhanced its efficiency.

\section{Ectopic bone formation of the rhBMP-2-loaded scaffolds}

Both the CPC/rhBMP-2 and CPC-AH/rhBMP-2 samples successfully induced ectopic bone formation, and the apparent distinction in sizes of the ectopic bone at weeks 2 and 4 between those groups was expressed by the digital and Micro-CT images (Fig. 8A). The size of new bone induced by CPC-AH/rhBMP-2 was larger than that by the CPC/rhBMP-2 sample even though it underwent the bone resorption at 4 weeks. Compared with the CPC/rhBMP-2 sample (Fig. 8B), the bone wet weight in CPC-AH/ rhBMP-2 was significantly increased by $11.44 \%$ at 2 weeks, while at 4 weeks the value was further elevated by $29.51 \%$. Moreover, at 2 weeks, the Micro-CT reconstructed images (Fig. 8A) of the CPC-AH/rhBMP-2 group showed more new bone formation and confluent ingrowths along with separated material fragments surrounded by bone tissue. More importantly, at week 4, more bone tissue (gray area) preservation and material (blue area) degradation was observed. The CPC-AH/ 
A

Oh

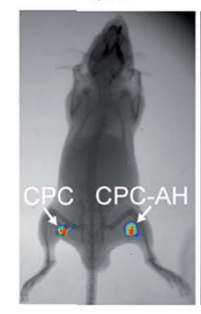

$24 h$

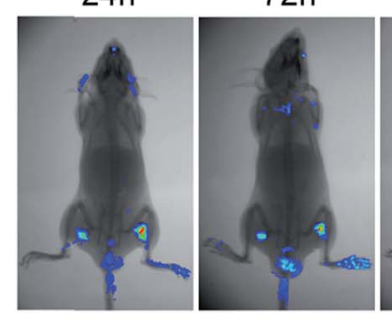

$168 \mathrm{~h}$
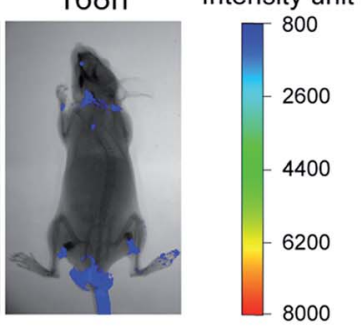

B

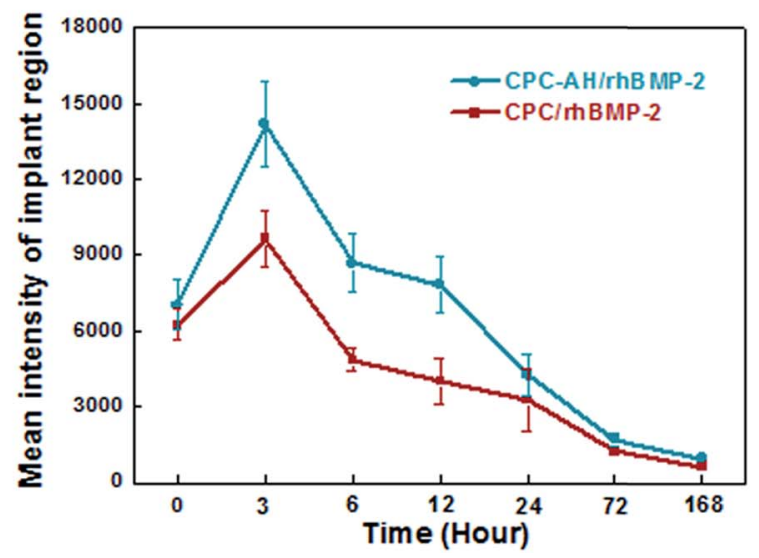

Fig. 7 (A) Real-time in vivo imaging of FITC-labeled rhBMP-2 released from the CPC (left hind leg) and CPC-AH (right hind leg) scaffolds at different time points after implantation. (B) Quantitative analysis of the mean fluorescent intensity at each time point for the target areas on both sides.

rhBMP-2 group possessed a higher ratio of new bone volume (BV) to total volume (TV) compared to the CPC/rhBMP-2 group, both at weeks 2 and 4 (Fig. 8C), indicating that CPC-AH facilitated new bone formation and bone preservation as well as accelerated the biodegradation rate of the scaffolds.

HE and Masson's trichrome staining methods were performed to compare the tissue morphology and composition of the ectopic bone at weeks 2 and 4. As shown in Fig. 8D, much more newly formed bones (NB) were found both at 2 or 4 weeks in the CPC-AH/rhBMP-2 group. Especially in undergoing the bone resorption at 4 weeks, a large amount of bone tissue was preserved in the CPC-AH/rhBMP-2 group, while there was a predominant amount of lipocytes (yellow circle, white cells) left in the CPC/rhBMP-2 group. The Masson trichrome staining reported a similar result, as shown in Fig. 8E. Compared to the

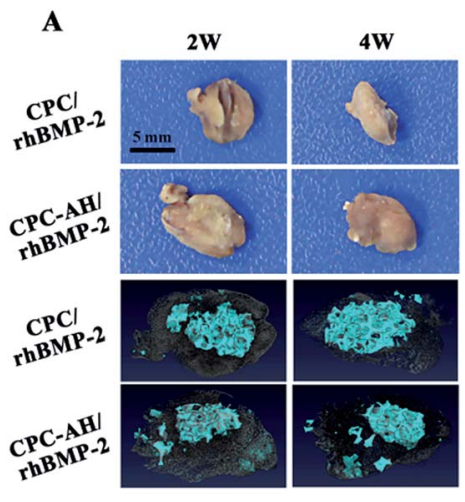

B
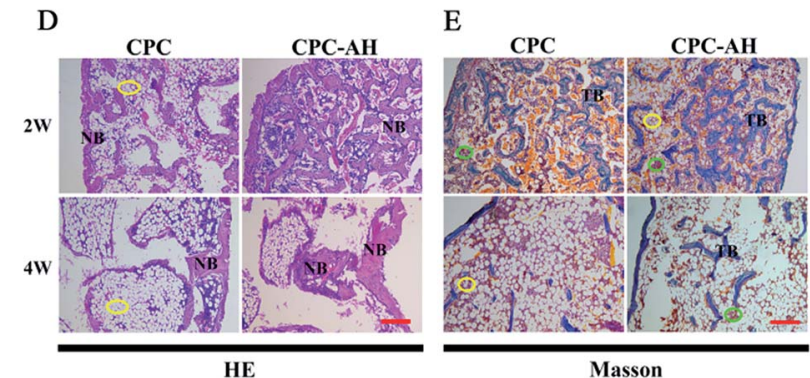

Fig. 8 Evaluation of ectopic bone formation at 2 and 4 weeks for the CPC/CPC-AH-loaded scaffolds. (A) Digital photographs (above) and 3D SR $\mu C T$ reconstructed images (below) of the ectopic bones. Black scale bar is $5 \mathrm{~mm}$. (B) Wet weight and (C) the percentage of bone volume (BV) to total volume (TV) of the ectopic bone $(n=5)$. ${ }^{*} p<0.05$. (D) $\mathrm{HE}$ and (E) Masson's trichrome staining of ectopic bone sections (NB: new bone, TB: trabecular bone, yellow circle: lipocytes, green circle: medulla ossium rubra). Red scale bar is $200 \mu \mathrm{m}$.

CPC/rhBMP-2 group, more newly formed ectopic bone was surrounded by bone marrow (yellow and green circle) at 2 weeks in the CPC-AH/rhBMP-2 group, then additionally, at 4 weeks, a continuous trabecula bone within richer medulla ossium rubra (TB, green circle) was clearly observed in the $\mathrm{CPC}-\mathrm{AH} /$ rhBMP-2 group, which still showed a higher content of bone tissues than in the CPC/rhBMP-2 group.

The greater area of integrated bone tissue structure formed in the CPC-AH/rhBMP-2 group pronouncedly demonstrated that the CPC-AH delivery system not only prolonged the release period of rhBMP-2, but also sustained the concentration of rhBMP-2 around the defect area, resulting in a higher osteogenetic efficiency of rhBMP-2 in vivo.

\section{Discussion}

The rapid and high-quality bone regeneration of defects remains a challenge in clinics worldwide. ${ }^{1,2}$ BMP-2 is approved as the most potent bone inductor and plays a significant role in osteoinduction and bone morphogenesis regulation, but its clinical use is currently limited due to its short half-life and offtarget release. Therefore, it is urgent to propose or optimize a localized and sustainable delivery of rhBMP-2 using new carrier materials to overcome the risks associated with supraphysiological doses usage. ${ }^{6,7}$ Bearing this in mind, a kind of novel delivery system was developed to tether rhBMP-2 on to 
a CPC substrate via AH molecule to concurrently satisfy both a high bioactive immobilization and the long-term release of rhBMP-2 for advanced local bone regeneration. With this representative, namely clinically widely-used calcium phosphate, the results found here may be of great value in the design and development of rhBMP-2-based superior bone regeneration materials.

In order to accomplish this, heparin was first activated by EDC to conjugate with alendronate. The resultant $\mathrm{AH}$ was adsorbed onto CPC and further tethered rhBMP-2 via a lyophilization fabrication process. As expected, our data clearly showed the prominent effect of the CPC-AH scaffold compared to the non-modified CPC sample on the release and osteoinduction of rhBMP-2, with evidence that the CPC-AH scaffold exhibited a lower burst release during a longer period $(40 \%$ in $168 \mathrm{~h}$ for CPC-AH vs. $40 \%$ in $10 \mathrm{~h}$ for CPC, Fig. 5B), enhanced ALP activity (Fig. 6), and promoted osteogenesis (Fig. 8) in vitro and in vivo.

As is well known, the key for effectively rhBMP-2-induced bone regeneration lies in localization and continuous osteogenic stimulation at defected sites ${ }^{\mathbf{4 1}}$ From this viewpoint, one major advantage of this delivery system is that this novel $\mathrm{AH}$ based tethering system could support a sustainably controlled release of rhBMP-2 as illustrated in the in vitro and in vivo release profiles (Fig. 5B and 7A) while maintaining high bioactivity locally as demonstrated by the enhanced alkaline phosphatase activity and ectopic bone formation after implantation in vivo (Fig. 6 and 8). To the best of our knowledge, BMPs often are loaded and delivered via physical and chemical absorption. ${ }^{42}$ The typical physical absorption, even applied in the FDAapproved collagen sponge, is accompanied by a clear disadvantage of burst and uncontrolled release that means the clinical need is unmet. ${ }^{22}$ Another strategy based on chemically binding BMP-2 onto the surface of scaffolds could help to prolong the exposure time of BMP-2, but it is severely limited by the loss of BMP-2 bioactivity. ${ }^{43}$ Fortunately, the tethering structure in this developed system builds the perfect balance between those two protocols and obtains a long-term controlled release with a high osteogenic activity of BMP-2.

Considering the fact that bone repair can be accelerated under the condition of local administration and a longretention time of $\mathrm{BMP}-2$, alendronate and heparin were combined to tether rhBMP-2 onto the surface of CPC to prolong exposure and regulate release in the target site. We give the credit for this to the tethering structure that serves as an intermediate junction between the rhBMP-2 and CPC substrate. As we know, the final hydration product of CPC, a mixture comprising equimolar tetracalcium phosphate (TECP) and dicalcium phosphate anhydrous (DCPA), is hydroxyapatite with abundant calcium ions, which can easily and strongly interact with phosphate anions of alendronate molecules. ${ }^{44}$ Meanwhile, in the terms of another end, rhBMP- 2 with positive charge was strongly bound to heparin with the negatively charged sulfate groups in a reversible manner. As a matter of fact, there is sufficient evidence that the heparin can locally immobilize a class of positively charged growth factors, and can exist both as free chains or proteoglycans. ${ }^{45}$ With the strong binding capacities between $\mathrm{AH}$ and $\mathrm{CPC}$ as well as $\mathrm{AH}$ and rhBMP-2, this new tethering delivery system effectively suppressed the free diffusion and ensured a continuously high concentration of rhBMP-2 at the site of interest.

Furthermore, this AH-based tethering system also represented another significant advantage in preserving and retaining the rhBMP-2-induced osteogenic capacity based on the results from the in vitro ALP activity and the ectopic bone formation assay. We believe there are two essential factors responsible for this bioactivity maintenance. First, the tethering structure prevents direct contact between rhBMP-2 and the substrate, which often easily results in denaturation, as found in other chemical adsorbed delivery systems. Second, it is the heparin that can supply high bioactivity immobilization and an appropriate environment for local bone regeneration. Heparin was proved to interact with various heparin-binding growth factors, such as the transforming growth factor b (TGF-b), vascular endothelial growth factor (VEGF), fibroblast growth factors (FGFs), insulin-like growth factors (IGFs), and bone morphogenetic proteins (BMPs), in a non-covalent and reversible way, ensuring a minimal impact on their structure during binding. ${ }^{45}$ Previous studies have proved that the addition of heparin to biomaterials contributes to improving growth factor retention and BMP-2-induced osteogenesis. ${ }^{37,46}$ Moreover, it is accepted that heparin itself may play an essential role in the preservation and presentation of bound proteins through electrostatic interactions. ${ }^{45,47}$ As a result, the AH-tethered rhBMP-2 upon CPC with desirable conformation exhibited excellent bioactivity.

In the bone regeneration treatment, the growth factors delivery system should not only act as a carrier to transport and control the release of growth factors in the target site under a modulated rate and dose, but it should also be responsible for preserving the bioactivities of the growth factors before coming into effect. Consistent with the previous reports and our hypothesis, the results from this work highlighted the outstanding properties of the fabricated CPC-AH scaffold in terms of the local immobilization, sustainable delivery, and
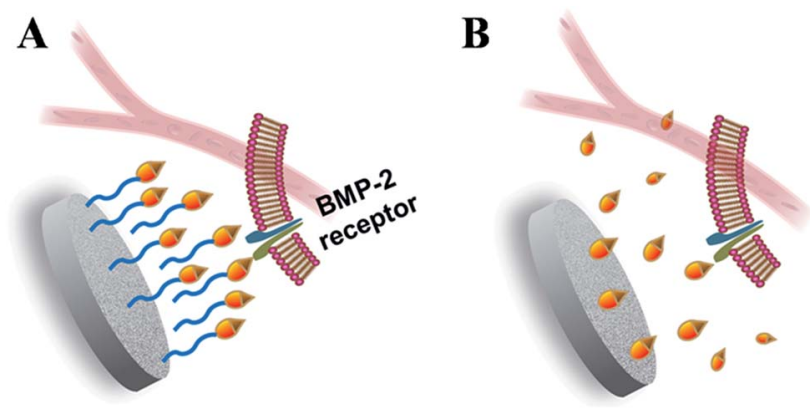

Fig. 9 Schematic diagram of the tethering effect of the $\mathrm{CPC}-\mathrm{AH}$ scaffold on the controlled release of rhBMP-2. Compared with the free diffusion of rhBMP-2 from (B) the CPC scaffold, the (A) CPC-AH scaffold effectively immobilized the rhBMP-2 in target size and guaranteed the free-like state of the protein interacting with its receptor. As a result, the locally sustainable concentration and high bioactivity of rhBMP-2 leads to more efficient bone regeneration. 
bioactive maintenance of rhBMP-2. In particular, the AH tethering structure played a critical role in the delivery process to warrant that rhBMP-2 could complete receptor binding and signaling on a cell surface in an immobilized but free-like state during a sufficient period to promote the local osteoinductive differentiation (Fig. 9).

\section{Conclusion}

RhBMP-2 was tethered onto the surface of calcium phosphate cement (CPC) via the alendronate-heparin (AH) compound, which exhibited strong binding between CPC and rhBMP-2. The release of rhBMP-2 was greatly reduced and thus the localized concentration at the target site was significantly retained. Meanwhile, substantial osteogenic differentiation was confirmed on the AH-tethered rhBMP-2 scaffold, which was attributed to the biologic functions of heparin. With the localized, long-term, and effective osteogenic induction, bone regeneration was promoted. This novel concept of tethering onto the scaffold provides a valuable strategy for the highly effective immobilization of rhBMP-2, and may be a promising method of delivering and for spatially retaining growth factors for a variety of applications.

\section{Acknowledgements}

The authors wish to express their gratitude to the financial supports from the National Natural Science Foundation of China (No. 31330028 and 31470924), and the 111 Project (B14018).

\section{References}

1 Y. M. Kolambkar, J. D. Boerckel, K. M. Dupont, M. Bajin, N. Huebsch, D. J. Mooney, D. W. Hutmacher and R. E. Guldberg, Bone, 2011, 49, 485-492.

2 J. M. Holzwarth and P. X. Ma, Biomaterials, 2011, 32, $9622-$ 9629.

3 G. Zimmermann and A. Moghaddam, Injury, 2011, 42(2), S16-S21.

4 Y. C. Chen, T. P. Sun, C. T. Su, J. T. Wu, C. Y. Lin, J. Yu, C. W. Huang, C. J. Chen and H. Y. Chen, ACS Appl. Mater. Interfaces, 2014, 6, 21906-21910.

5 S. K. Perikamana, J. Lee, T. Ahmad, Y. Jeong, D. G. Kim, K. Kim and H. Shin, ACS Appl. Mater. Interfaces, 2015, 7, 8798-8808.

6 R. C. de Guzman, J. M. Saul, M. D. Ellenburg, M. R. Merrill, H. B. Coan, T. L. Smith and M. E. Van Dyke, Biomaterials, 2013, 34, 1644-1656.

7 S. Suliman, Z. Xing, X. Wu, Y. Xue, T. O. Pedersen, Y. Sun, A. P. Doskeland, J. Nickel, T. Waag, H. Lygre, A. FinneWistrand, D. Steinmuller-Nethl, A. Krueger and K. Mustafa, J. Controlled Release, 2015, 197, 148-157.

8 X. Shen, Y. Zhang, Y. Gu, Y. Xu, Y. Liu, B. Li and L. Chen, Biomaterials, 2016, 106, 205-216.
9 J. D. Boerckel, Y. M. Kolambkar, K. M. Dupont, B. A. Uhrig, E. A. Phelps, H. Y. Stevens, A. J. Garcia and R. E. Guldberg, Biomaterials, 2011, 32, 5241-5251.

10 L. Cao, J. Wang, J. Hou, W. Xing and C. Liu, Biomaterials, 2014, 35, 684-698.

11 S. E. Bae, J. Choi, Y. K. Joung, K. Park and D. K. Han, J. Controlled Release, 2012, 160, 676-684.

12 T. Gao, W. Cui, Z. Wang, Y. Wang, Y. Liu, P. S. Malliappan, Y. Ito and P. Zhang, RSC Adv., 2016, 6, 20202-20210.

13 F. Danhier, E. Ansorena, J. M. Silva, R. Coco, A. Le Breton and V. Préat, J. Controlled Release, 2012, 161, 505-522.

14 M. Bouyer, R. Guillot, J. Lavaud, C. Plettinx, C. Olivier, V. Curry, J. Boutonnat, J. L. Coll, F. Peyrin, V. Josserand, G. Bettega and C. Picart, Biomaterials, 2016, 104, 168-181.

15 X. Qu, F. He, H. Tan, Y. Yu, A. Axrap, M. Wang, K. Dai, Z. Zhang, F. Yang, S. Wang, J. Kohn and C. Liu, J. Mater. Chem. B, 2016, 4, 4901-4912.

16 C. Y. Chien and W. B. Tsai, ACS Appl. Mater. Interfaces, 2013, 5, 6975-6983.

17 A. M. Peterson, C. Pilz-Allen, T. Kolesnikova, H. Mohwald and D. Shchukin, ACS Appl. Mater. Interfaces, 2014, 6, 1866-1871.

18 X. Fang, J. Xie, L. Zhong, J. Li, D. Rong, X. Li and J. Ouyang, J. Mater. Chem. B, 2016, 4, 1070-1080.

19 S. Lu, J. Lam, J. E. Trachtenberg, E. J. Lee, H. Seyednejad, J. J. van den Beucken, Y. Tabata, M. E. Wong, J. A. Jansen, A. G. Mikos and F. K. Kasper, Biomaterials, 2014, 35, 88298839.

20 A. Neumann, A. Christel, C. Kasper and P. Behrens, RSC Adv., 2013, 3, 24222-24230.

21 Q. Gan, J. Zhu, Y. Yuan, H. Liu, J. Qian, Y. Li and C. Liu, J. Mater. Chem. B, 2015, 3, 2056-2066.

22 M. Geiger, Adv. Drug Delivery Rev., 2003, 55, 1613-1629.

23 E. J. Carragee, E. L. Hurwitz and B. K. Weiner, Spine Journal, 2011, 11, 471-491.

24 C. A. Tannoury and H. S. An, Spine Journal, 2014, 14, 552559.

25 S. Shiels, S. Oh, C. Bae, T. Guda, B. Singleton, D. D. Dean, J. C. Wenke, M. R. Appleford and J. L. Ong, J. Biomed. Mater. Res., Part B, 2012, 100, 1782-1791.

26 T. Crouzier, F. Sailhan, P. Becquart, R. Guillot, D. LogeartAvramoglou and C. Picart, Biomaterials, 2011, 32, 7543-7554.

27 R. Derynck and Y. E. Zhang, Nature, 2003, 425, 577-584.

28 V. Rosen, Cytokine Growth Factor Rev., 2009, 20, 475-480.

29 J. S. Park, S. W. Yi, H. J. Kim, S. M. Kim and K. H. Park, ACS Appl. Mater. Interfaces, 2016, 8, 30387-30397.

30 J. Massague, J. Biol. Chem., 1990, 265, 21393-21396.

31 S. M. Shiels, K. D. Solomon, M. Pilia, M. R. Appleford and J. L. Ong, J. Biomed. Mater. Res., Part A, 2012, 100, 3117-3123.

32 J. H. Lee, J. H. Park, Y. R. Yun, J. H. Jang, E. J. Lee, W. Chrzanowski, I. B. Wall and H. W. Kim, J. Mater. Chem. $B, 2013,1,2731-2741$.

33 H. W. Liu, C. H. Chen, C. L. Tsai, I. H. Lin and G. H. Hsiue, Tissue Eng., 2007, 13, 1113-1124.

34 G. S. Lee, J. H. Park, U. S. Shin and H. W. Kim, Acta Biomater., 2011, 7, 3178-3186. 
35 C. Canal, S. Gallinetti and M. P. Ginebra, Plasma Processes Polym., 2014, 11, 694-703.

36 X. Shi, L. Ren, M. Tian, J. Yu, W. Huang, C. Du, D. A. Wang and Y. Wang, J. Mater. Chem., 2010, 20, 9140-9148.

37 J. L. Roam, P. K. Nguyen and D. L. Elbert, Biomaterials, 2014, 35, 6473-6481.

38 J. Zhang, H. Zhou, K. Yang, Y. Yuan and C. Liu, Biomaterials, 2013, 34, 9381-9392.

39 A. J. Melchiorri, N. Hibino, T. Yi, Y. U. Lee, T. Sugiura, S. Tara, T. Shinoka, C. Breuer and J. P. Fisher, Biomacromolecules, 2015, 16, 437-446.

40 A. Dolatshahi-Pirouz, T. Jensen, M. Foss, J. Chevallier and F. Besenbacher, Langmuir, 2009, 25, 2971-2978.
41 B. B. Seo, H. Choi, J. T. Koh and S. C. Song, J. Controlled Release, 2015, 209, 67-76.

42 K. Lee, E. A. Silva and D. J. Mooney, J. R. Soc., Interface, 2011, 8, 153-170.

43 L. Tao, J. Liu, J. Xu and T. P. Davis, Org. Biomol. Chem., 2009, 7, 3481-3485.

44 Z. Shen, T. Yu and J. Ye, Mater. Sci. Eng., C, 2014, 42, 303311.

45 M. H. Hettiaratchi, T. Miller, J. S. Temenoff, R. E. Guldberg and T. C. McDevitt, Biomaterials, 2014, 35, 7228-7238.

46 O. Jeon, C. Powell, L. D. Solorio, M. D. Krebs and E. Alsberg, J. Controlled Release, 2011, 154, 258-266.

47 W. J. Kuo, M. A. Digman and A. D. Lander, Mol. Biol. Cell, 2010, 21, 4028-4041. 